\title{
Crescimento de plantas jovens de Tabebuia aurea (Manso) Benth. \& Hook. f. ex S. Moore submetidas a estresse hídrico ${ }^{1}$
}

\author{
Edna Lopes Cabral ${ }^{2,3}$, Dilosa Carvalho de Alencar Barbosa² e Eliana Akie Simabukuro ${ }^{2}$
}

Recebido em 25/09/2002. Aceito em 17/08/2003

\begin{abstract}
RESUMO - (Crescimento de plantas jovens de Tabebuia aurea (Manso) Benth. \& Hook. f. ex S. Moore submetidas a estresse hídrico). Tabebuia aurea (Manso) Benth. \& Hook. f. ex S. Moore pertence à família Bignoniaceae e ocorre nas margens dos rios temporários do Nordeste semi-árido. O crescimento das plantas jovens foi acompanhado em casa de vegetação por quatro meses e foi analisado em função do alongamento $(\mathrm{cm})$, massa da matéria seca $(\mathrm{g})$ e densidade estomática $\left(\mathrm{mm}^{2}\right)$. Trinta dias após a semeadura, as plantas foram submetidas a três tratamentos hídricos: 100, 50 e $25 \%$ da capacidade de campo (cc). A densidade estomática foi verificada mensalmente nas regiões apical, mediana e basal da folha. Utilizou-se impressão em esmalte e a contagem foi efetuada em área de $1 \mathrm{~mm}^{2}$. O tratamento de $25 \%$ cc propiciou maior redução do crescimento. Nesse tratamento, a razão de crescimento (cm) parte subterrânea/aérea foi de 2:1 até os 60 dias; o número médio de folhas produzidas reduziu a partir dos 90 dias, e a área foliar média, dos 30 aos 120 dias de experimento. Em todos os tratamentos, evidenciou-se o efeito do estresse na massa da matéria seca (g) a partir dos 90 dias. O maior percentual de biomassa foi alocado para a parte aérea até os 90 dias e aos 120 dias para a parte subterrânea. T. aurea é hipoestomática, com estômatos do tipo anomocítico e densidade estomática variando de 119,63 a 155,19 estômatos por mm² $^{2}$.
\end{abstract}

Palavras-chave: Tabebuia aurea, crescimento, área foliar, alocação de biomassa, densidade estomática

\begin{abstract}
Growth of young plants of Tabebuia aurea (Manso) Benth. \& Hook. f. ex S. Moore under water stress). Tabebuia aurea (Manso) Benth. \& Hook. f. ex S. Moore, a member of the family Bignoniaceae, is usually found along the margins of temporary rivers in the semiarid region of Northeastern Brazil. Growth of young plants was studied for four months in a greenhouse, with respect to elongation $(\mathrm{cm})$, dry matter weight $(\mathrm{g})$ and stomatal density $\left(\mathrm{mm}^{2}\right)$. Thirty days after sowing, the young plants were submitted to three water treatments: 100,50 and $25 \%$ of field capacity. Leaf stomatal density at the apical, median and basal portions was recorded monthly. Stomata impression in enamel was used and countings were performed in a $1 \mathrm{~mm}^{2}$ area. The $25 \%$ field capacity treatment had the most negative effects on growth. Under this treatment, root system/aerial portion growth ratio (cm) was of a 2:1 magnitude up to 60 days; average leaf number was reduced from 90 days on, and average leaf area was reduced from 30 to 120 days of the trial. For all treatments, stress effect was evident on dry matter weight (g) from 90 days on. Higher biomass allocation percentage was observed on young plant aerial portion up to 90 days and on root system at 120 days. T. aurea is hypostomatic, with anomocytic stomates. Stomatal density varied from 119,63 to $155,19 / \mathrm{mm}^{2}$.
\end{abstract}

Key words: Tabebuia aurea, growth, leaf area, biomass allocation, stomatal density

\section{Introdução}

A região semi-árida do Nordeste brasileiro corresponde a $910.000 \mathrm{~km}^{2}$, ocupando cerca de $11 \%$ do território nacional, sendo a Caatinga o tipo vegetacional que a caracteriza (Rizzini 1997). Trata-se de um dos ecossistemas mais ameaçado e menos conhecido do ponto de vista científico no Brasil, com riqueza de espécies, possuindo potencial madeireiro, medicinal, alimentício e ornamental (Mansur \& Barbosa 2000).

Dentre as espécies que ocorrem neste ecossistema destaca-se Tabebuia aurea (Manso) Benth. \& Hook f. ex S. Moore, pertencente à Família Bignoniaceae. Árvore de 12 a $20 \mathrm{~m}$ alt., com tronco tortuoso, revestido por casca grossa, apresentando folhas opostas, compostas e subcoriáceas, frutos do tipo síliqua, com cerca de 80 sementes, achatadas, aladas. É comum às margens dos rios temporários do Nordeste semiárido e integra também a flora dos Cerrados e Cerradões de quase todo o Brasil. Planta de múltiplas utilidades na construção civil, na medicina popular e ornamental, sendo indicada para trabalhos de reflorestamento em áreas de baixa pluviosidade (Corrêa 1984; Andrade-Lima 1989; Lorenzi 1992; Gentry 1992).

\footnotetext{
1 Parte da Dissertação de Mestrado do primeiro Autor

2 Departamento de Botânica, Universidade Federal de Pernambuco, Av. Prof. Moraes Rego s/n, Cidade Universitária, CEP 50560-901, Recife, PE, Brasil

3 Autor para correspondência: ednacabral@yahoo.com.br
} 
Diversos fatores influenciam no crescimento das plantas, dentre eles pode-se citar a disponibilidade de nutrientes, luz e água (Sassaki \& Felippe 1992; Brighenti et al. 1993; Perez 1995; Teixeira et al. 1997; Nardoto et al. 1998; Andrade et al. 1999). A ausência deste último é o fator que condiciona a vegetação da Caatinga, pois o déficit hídrico durante grande parte do ano faz com que a fisionomia e a flora variem grandemente (Sampaio 1995).

Como são evidentes os efeitos do ambiente no crescimento, no desenvolvimento e na produtividade vegetal, é essencial a avaliação dos efeitos desses fatores na fisiologia do crescimento das espécies de importância econômica e ecológica.

Nas últimas décadas vem se intensificando na Caatinga estudos ecofisiológicos da germinação e do crescimento em condições de laboratório, casa de vegetação e in loco (Barbosa 1991; Barbosa \& Prado 1991; Gama 1992; Barros \& Barbosa 1995; Barbosa et al. 2000). No entanto, ainda são poucos os trabalhos com as espécies lenhosas desse ecossistema. Experimentos de estresse hídrico durante o crescimento inicial demonstraram alta tolerância quando as plantas jovens foram submetidas ao tratamento de $50 \%$ da capacidade de campo (Barros \& Barbosa 1995; Mansur \& Barbosa 2000; Moraes 2002; Silva 2002).

Considerando a importância de T. aurea e a necessidade de melhor compreender a sua sobrevivência em ambientes naturais, sob condições estressantes, este trabalho teve como objetivos determinar quantitativamente o alongamento $(\mathrm{cm}), \mathrm{o}$ peso a seco $(\mathrm{g})$ e a densidade estomática das plantas jovens em casa de vegetação, visando investigar os mecanismos adaptativos desta espécie, principalmente aqueles relacionados à baixa disponibilidade hídrica até quatro meses de idade. Informações básicas sobre esses processos fisiológicos são importantes para fornecer subsídios quanto à irrigação das mudas nos programas de reflorestamento para a região semi-árida nordestina.

\section{Material e métodos}

Os frutos de Tabebuia aurea foram coletados na população presente na Caatinga da região de Xingó, Fazenda Brejo, Município de Canindé do São Francisco, SE, situada entre os meridianos de $9^{\circ} 30^{\prime} \mathrm{e}$ $10^{\circ} 00^{\prime} \mathrm{S}$ e os paralelos de $37^{\circ} 30^{\prime}$ e $38^{\circ} 00^{\prime} \mathrm{W}$. O material botânico foi coletado e depositado no Herbário UFP da Universidade Federal de Pernambuco (N. 31042). Os experimentos foram conduzidos em casa de vegetação do Departamento de Botânica, Universidade Federal de Pernambuco, no período de fevereiro a junho/2001.

Os frutos e as unidades de dispersão foram secos ao ar livre e armazenadas em câmara fria $\left(15^{\circ} \mathrm{C}\right.$ e $40 \%$ de UR) na Empresa Pernambucana de Pesquisa Agropecuária (IPA), por dois meses. Foram selecionadas 400 unidades de dispersão, que foram distribuídas em bandejas plásticas de $30 \mathrm{~cm} \times 50 \mathrm{~cm}$, contendo areia lavada suficiente para cobri-las $( \pm 4 \mathrm{~cm})$ e após dez dias de germinadas, quando as plântulas estavam com aproximadamente $4 \mathrm{~cm}$ alt. e o $1^{\circ}$ par de folhas, foram selecionadas 144 e transplantadas para 48 sacos de polietileno de cor preta, preenchidos com solo do local de coleta das unidades de dispersão, de textura franco-arenosa (Tab. 1 e 2). Foram utilizados 24 sacos com capacidade para $6 \mathrm{~kg}$ de solo $(30 \mathrm{~cm}$ alt. e $15 \mathrm{~cm}$ diâm.), para os dois primeiros meses ( 30 e 60 dias) de avaliação, e 24 sacos com capacidade para $12 \mathrm{~kg}$ de solo ( $40 \mathrm{~cm}$ alt. e $25 \mathrm{~cm}$ diâm.), para os dois últimos meses (90 e 120 dias), recebendo cada um três plântulas. Para minimizar a evaporação o solo foi coberto com uma superfície de polietileno de cor preta. A distribuição das plantas na bancada em casa de vegetação foi inteiramente casualizada.

O solo utilizado foi seco ao ar livre, peneirado e a capacidade de campo foi determinada segundo Nogueira (1997). Trinta dias após o transplantio, iniciouse a diferenciação dos tratamentos hídricos conduzidos segundo método adotado por Barros \& Barbosa (1995). As plantas jovens foram mantidas por 120 dias com

Tabela 1. Análise física de um solo de Caatinga coletado na região de Xingó, Fazenda Brejo, Município de Canindé do São Francisco (SE) e utilizado nos experimentos.

\begin{tabular}{|c|c|c|c|c|c|c|c|c|c|c|}
\hline \multicolumn{4}{|c|}{ Granulometria (\%) } & \multicolumn{2}{|c|}{ Densidade } & \multirow[t]{2}{*}{ Classe textural } & \multicolumn{3}{|c|}{ Umidade (\%) } & \multirow{2}{*}{$\begin{array}{c}\text { Água } \\
\text { disponível }(\%)\end{array}$} \\
\hline Areia grossa & Areia fina & Silte & Argila & Dap & $\mathrm{Dr}$ & & Residual & $0,33 \mathrm{~atm}$ & $15 \mathrm{~atm}$ & \\
\hline 6 & 53 & 27 & 14 & 1,48 & 2,71 & FA & 1,00 & 20,26 & 4,57 & 15,69 \\
\hline
\end{tabular}

Análise feita pelo Laboratório de Solos, IPA. 
Tabela 2. Análise química de um solo de Caatinga coletado na região de Xingó, Fazenda Brejo, Município de Canindé do São Francisco (SE) e utilizado nos experimentos.

\begin{tabular}{cccccc}
\hline $\begin{array}{c}\mathrm{pH} \\
\left(\mathrm{H}_{2} \mathrm{O}\right)\end{array}$ & $\mathrm{Ca}$ & $\mathrm{Mg}$ & $\begin{array}{c}\mathrm{Na} \\
\mathrm{cmolc} / \mathrm{dm}^{3}\end{array}$ & $\mathrm{~K}$ & $\mathrm{H}$ \\
\hline 5,75 & 3,60 & 1,00 & 0,14 & 0,11 & 1,07 \\
\hline
\end{tabular}

Análise feita pelo Laboratório de Solos, IPA.

suprimento diário de água até 100,50 e $25 \%$ da capacidade de campo. Diariamente e sempre no mesmo horário, eram pesados os sacos plásticos com as plantas, em uma balança elétrica com duas casas decimais, para verificação da quantidade de água consumida e, posteriormente, adicionados os volumes a fim de manter a capacidade hídrica de cada tratamento (Moraes 2002).

Paralelamente, monitorou-se a temperatura $\left({ }^{\circ} \mathrm{C}\right)$ e a umidade relativa do ar (UR \%) na casa de vegetação através de termohigrógrafo (Tab. 3). A temperatura máxima variou de 35,4 a $37,2^{\circ} \mathrm{C}$, a mínima de 22,6 a $24,0^{\circ} \mathrm{C}$, e a UR 97 a $100 \%$ (máxima) e 46 a $52 \%$ (mínima).

Após 30, 60, 90 e 120 dias de suprimento hídrico diferenciado, 4 sacos (totalizando 12 plantas) para cada tratamento foram selecionados a fim de realizar a medida dos comprimentos das partes aérea e subterrânea $(\mathrm{cm})$, pesagem da matéria seca $(\mathrm{g})$, contagem do número de folhas e cálculos da área foliar $\left(\mathrm{cm}^{2}\right)$ e da densidade estomática $\left(\mathrm{mm}^{2}\right)$.

Para retirada das raízes, sem que estas fossem danificadas, os sacos foram rompidos verticalmente e a terra removida com água corrente, para evitar a perda de fragmentos da raiz.

Os comprimentos dos caules e das raízes foram determinados com auxílio de trena. Para a medida do

Tabela 3. Dados climáticos registrados na casa de vegetação do Departamento de Botânica da Universidade Federal de Pernambuco, no período de janeiro a junho/2001.

\begin{tabular}{|c|c|c|c|c|c|c|}
\hline \multirow[t]{2}{*}{ Meses } & \multicolumn{3}{|c|}{ Temperatura do ar $\left({ }^{\circ} \mathrm{C}\right)$} & \multicolumn{3}{|c|}{ Umidade relativa do ar $(\%)$} \\
\hline & Máxima & Mínima & Média & Máxima & Mínima & Média \\
\hline Janeiro & 37,2 & 24,0 & 30,6 & 97 & 48 & 72,5 \\
\hline Fevereiro & o 36,6 & 23,2 & 29,9 & 98 & 46 & 72,0 \\
\hline Março & 35,4 & 22,6 & 29,0 & 100 & 52 & 76,0 \\
\hline Abril & 36,4 & 24,0 & 30,2 & 100 & 53 & 76,5 \\
\hline Maio & 35,6 & 23,2 & 29,4 & 100 & 48 & 74,0 \\
\hline Junho & 35,8 & 24,0 & 29,9 & 98 & 52 & 75,0 \\
\hline
\end{tabular}

caule, considerou-se a distância entre o colo e o ponto de inserção das folhas mais jovens.

Os valores médios de área foliar foram obtidos por intermédio de todas as folhas em imagens digitalizadas em scanner de mesa, utilizando-se o programa de análise de imagem Image Tool (UTHSCSA 1997).

Depois de realizadas as mensurações, as plantas separadas em raízes, cotilédones, caules e folhas, foram submetidas à secagem em estufa a $105^{\circ} \mathrm{C}$, durante 72 horas. As determinações do peso da matéria seca dos diferentes órgãos foram obtidas em balança analítica (AND/HR200).

Para o estudo da densidade estomática utilizou-se o "método de réplica", que consiste em cobrir as superfícies foliares com uma camada de esmalte incolor, que, ao se solidificar, retrata a imagem da superfície da folha. Depois de secas, as camadas de esmalte são removidas e montadas em lâminas histológicas. As camadas de esmalte foram colocadas em uma folha do terceiro par (do ápice para a base), nas superfícies adaxial e abaxial e nas regiões apical, mediana e basal. Efetuou-se quatro repetições, totalizando quatro folhas para cada um dos três tratamentos. As lâminas foram observadas diretamente em microscópio óptico binocular, com aumento de 100 vezes, em uma área de $1 \mathrm{~mm}^{2}$, com auxílio de uma ocular milimetrada. Para cada região da folha foram realizadas quatro contagens, totalizando 16 por tratamento e região da folha.

Para as análises estatísticas os dados foram submetidos à análise de variância e as médias comparadas pelo teste de Tukey a 5\% de probabilidade, utilizando-se o programa SWNTIA (EMBRAPA 1996). As análises estatísticas foram aplicadas separadamente para os dois volumes de solo utilizados, $6 \mathrm{~kg}$ (30 e 60 dias) e $12 \mathrm{~kg}$ (90 e 120 dias), respectivamente.

\section{Resultados e discussão}

As sementes de Tabebuia aurea germinaram com 48 horas após o rompimento dos tegumentos pela radícula. As plântulas com cotilédones crassos de reserva são classificadas, segundo Garwood (1996), do tipo epígeo-carnosa. Os cotilédones foram libertados dos tegumentos em média no quarto dia após a germinação, adquiriram a coloração verde nos primeiros dias de crescimento e mantiveram-se aderidos às plantas até os 120 dias, quando o seu volume havia diminuído e apresentavam coloração marrom. Hamburgo-Alves (1975) e Nardoto et al. (1998), estudando plantas jovens de Jacaranda brasiliana 
(Lam.) Pers. (Bignoniaceae) e Kielmeyera coriaceae (Spr.) Mart. (Guttiferae), espécies lenhosas com sementes aladas ocorrentes no Cerrado, verificaram que as folhas cotiledonares permaneceram presas as plantas por 120 e 180 dias, respectivamente.

Souto (1996) constatou que a permanência dos cotilédones variou de 15 a 60 dias em seis espécies lenhosas de Leguminosae da Caatinga, assim discriminadas: Senna martiana (Benth.) Irwin \& Barneby, Senna spectabilis (Schrader) Irwin \& Barneby, Caesalpinia pyramidalis Tul., Caesalpinia ferrea Mart. ex Tul., Anadenanthera colubrina (Griseb.) Altschul. e Parapiptadenia zehntneri (Harm.) M.P. Lima \& Lima.

Por sua vez, Silva \& Silva (1974) e Feliciano (1989) constataram a persistência dos cotilédones de Spondias tuberosa Arruda e Schinopsis brasiliensis Engl., ambas Anacardiaceae e características da Caatinga, durante 60 e 180 dias, respectivamente. O maior tempo de manutenção registrado para as espécies lenhosas desse ecossistema foi 24 meses para Aspidosperma pyrifolium Mart. (Apocynaceae), segundo Valente \& Carvalho (1973).

Os cotilédones são importantes fontes de energia e responsáveis pelo crescimento inicial e sua remoção pode causar a redução de biomassa na planta jovem. Tal fato foi constatado por Sassaki \& Felippe (1992) em plantas jovens de Dalbergia miscolobium Benth. (Leguminosae) do Cerrado brasileiro.

Tabebuia aurea, na fase de planta jovem, apresenta heterofilia, ou seja folhas simples subcoriáceas, transformando-se em folhas do tipo palmaticompostas na fase adulta. Nesse trabalho, até os 120 dias foram evidenciadas folhas simples (Fig. 1). Melo (1995), analisando o desenvolvimento morfo-anatômico de $T$. aurea, registrou a presença de folhas simples na planta jovem até 24 meses de idade. Esse comportamento também foi evidenciado por Hamburgo-Alves (1975) para as plantas jovens de Jacaranda brasiliana, outra espécie de Bignoniaceae.

Crescimento das partes aérea e subterrânea - o estresse hídrico reduziu o crescimento $(\mathrm{cm})$ da parte aérea das plantas com $25 \%$ cc até os 120 dias (Fig. 2A). As plantas com 50\% cc diferiram apenas aos 90 dias. Resultado semelhante foi encontrado por Barbosa (1991) ao estudar o crescimento em alongamento $(\mathrm{cm})$ de Anadenanthera macrocarpa (Benth.) Brenan (Leguminosae - Mimosoideae), cultivada com e sem suprimento hídrico, durante 150 dias. A autora verificou que o estresse hídrico reduziu o crescimento do caule a partir dos 90 dias. Barros \& Barbosa (1995) constataram que o crescimento $(\mathrm{cm})$ da parte aérea de plantas jovens de Acacia farnesiana Willd. (Leguminosae - Mimosoideae) sem suprimento hídrico durante 90 dias, foi significativamente menor quando comparado aos tratamentos com suprimentos diários de $150 \mathrm{~mL}$ e $75 \mathrm{~mL}$ de água.

Quanto ao crescimento $(\mathrm{cm})$ da parte subterrânea (Fig. 2B), não houve diferença significativa entre os tratamentos até os 60 dias. As diferenças tornaram-se mais acentuadas nas plantas com $25 \% \mathrm{cc}$, que apresentaram os menores valores $31,56 \mathrm{~cm}$ e $31,86 \mathrm{~cm}$ aos 90 e 120 dias, respectivamente, quando comparadas com as plantas submetidas ao suprimento hídrico diário de $100 \%$ cc. O crescimento da parte subterrânea foi superior ao da parte aérea (Fig. 2A e 2B) nos três tratamentos do início ao final do experimento, ocorrendo nas plantas com $25 \%$ cc uma proporção de 2:1 aos 30 e 60 dias, fato não registrado aos 90 e 120 dias, cuja proporção foi da ordem de 1:1.
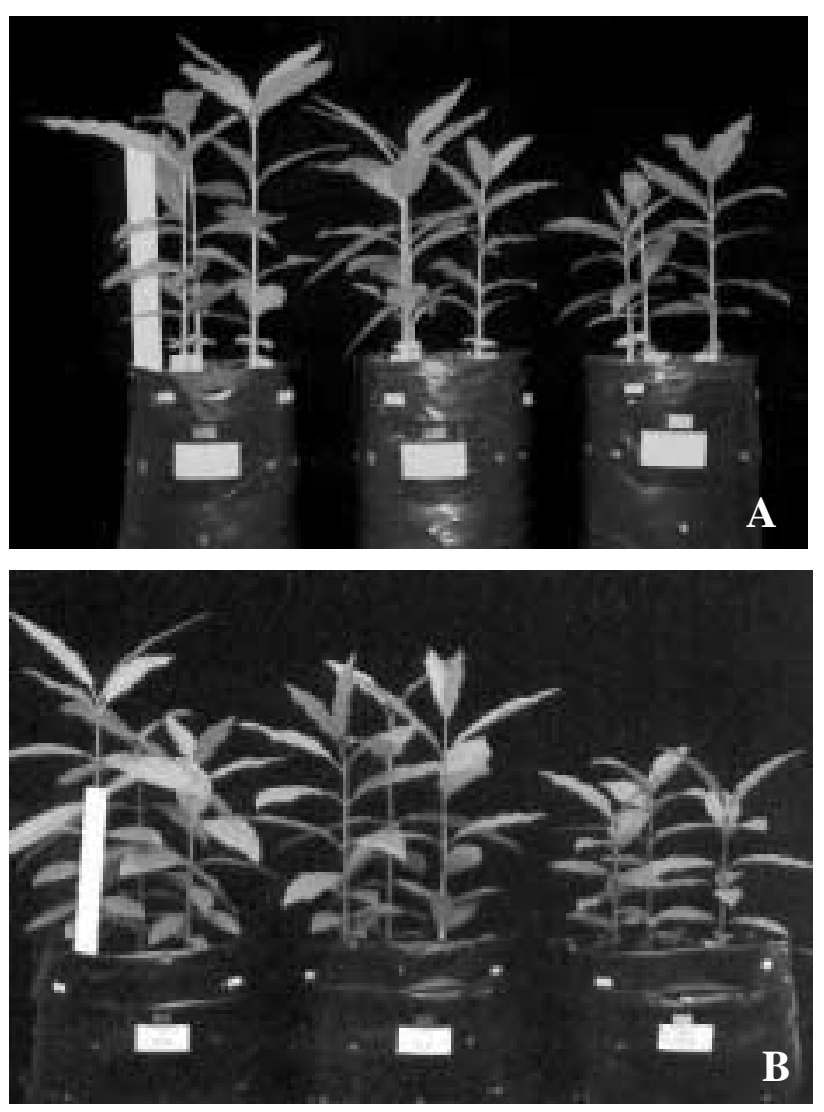

Figura 1. Aspecto das plantas jovens de Tabebuia aurea (Manso) Benth. \& Hook f. ex S. Moore, aos 30 (A) e 120 (B) dias de cultivo em casa de vegetação, apresentando folhas simples. Irrigadas com 100, 50 e $25 \%$ da capacidade de campo, seguindo a ordem da esquerda para direita. $($ Escala $=20 \mathrm{~cm})$. 

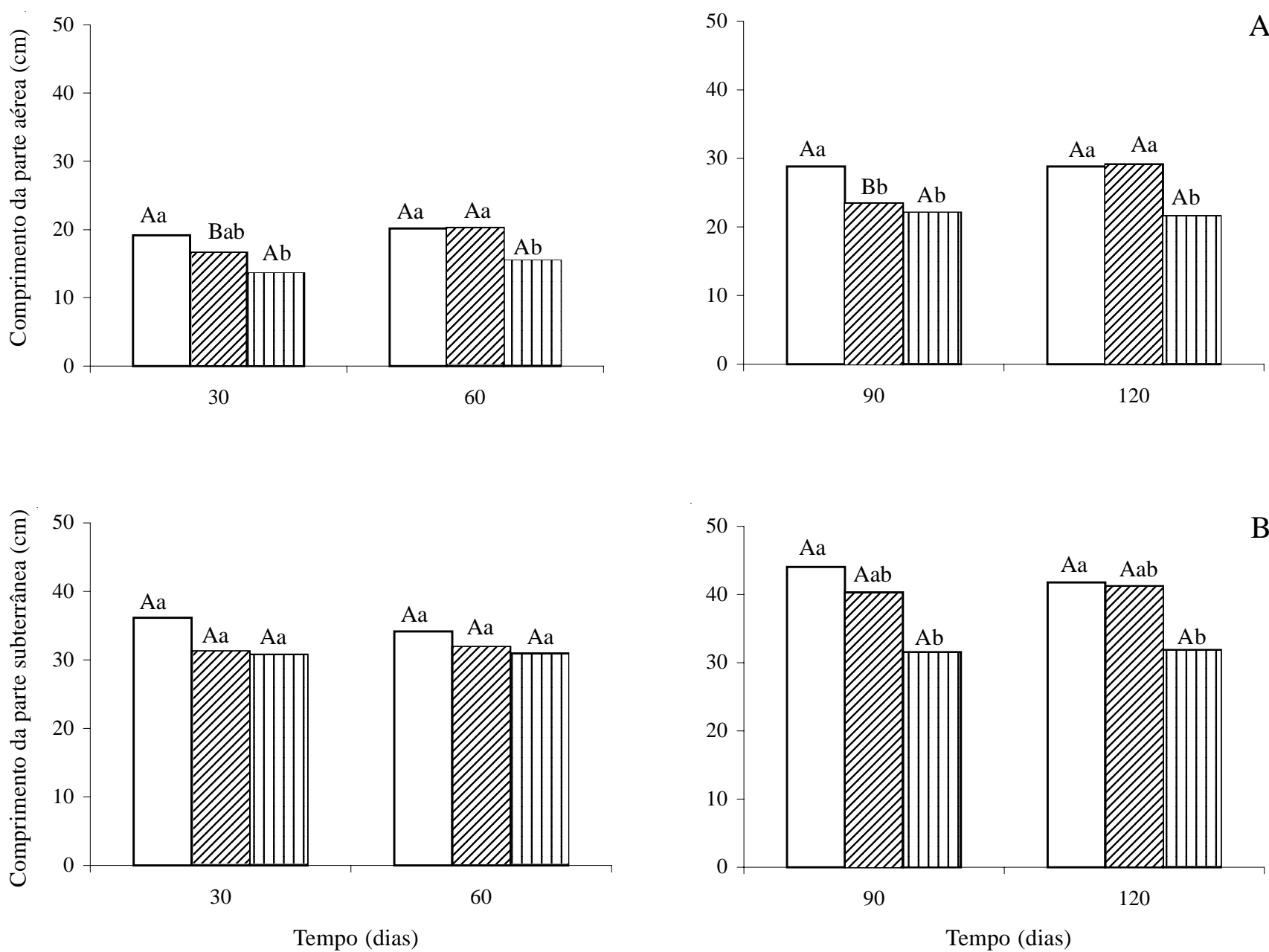

Figura 2. Comprimento médio das partes aérea (A) e subterrânea (B) das plantas jovens de Tabebuia aurea (Manso) Benth. \& Hook f. ex S. Moore, cultivadas em casa de vegetação sob três regimes hídricos: 100, 50 e $25 \%$ da capacidade de campo (cc). Análise estatística realizada separadamente para os dois volumes de solo utilizados: $6 \mathrm{~kg}$ (30 e 60 dias) e $12 \mathrm{~kg}$ (90 e 120 dias). Letras iguais não diferem entre os tratamentos (minúsculas) e períodos (maiúsculas) pelo teste de Tukey à $5 \%$ de probabilidade. $\square 100 \% \mathrm{cc} ; \square 50 \% \mathrm{cc} ; \square 25 \% \mathrm{cc}$.

O crescimento inicial da espécie $T$. aurea segue os padrões apresentados por algumas espécies da Caatinga e do Cerrado, este último ecossistema, com vegetação também sujeita a estresse hídrico, com uma nítida estação seca e outra chuvosa. A maior contribuição da parte subterrânea no comprimento total da planta jovem é observado na Caatinga, em Parkinsonia aculeata L. (Barbosa \& Prado 1991), Anadenanthera macrocarpa (Barbosa 1991) e Acacia farnesiana (Barros \& Barbosa 1995); e no Cerrado, em Andira humilis Mart. ex Benth. (Handro 1969), Kielmeyera coriacea (Spr.) Mart. (Arasaki \& Felippe 1991), Qualea cordata Spreng. (Godoy \& Felippe 1992), Qualea grandiflora Mart. (Paulilo et al. 1993; Paulilo \& Felippe 1994; 1995) e Solanum lycocarpum St. Hil. (Vidal et al. 1999). Esse comportamento, maior comprimento da parte subterrânea em relação à parte aérea é caráter adaptativo, comum às plantas submetidas a estresse hídrico, sendo vantajoso por permitir que as mesmas obtenham água mesmo depois da superfície do solo ter perdido a umidade durante a estação seca (Poggiani 1971; 1973; Hsiao 1973; Poggiani et al. 1981; Larcher 2000), fato este observado com mais freqüência no Cerrado, devido ao lençol freático ser mais profundo, em relação ao ecossistema Caatinga.

Número médio de folhas e área foliar média - Quanto ao número médio de folhas não foram registradas diferenças significativas entre os tratamentos até os 60 dias; no entanto, o tratamento de $25 \%$ cc em valores absolutos foi sempre inferior aos demais tratamentos. Esta redução foi mais acentuada após os 90 dias, quando os valores passaram a diferir significativamente nas plantas com $100 \%$ cc, e aos 120 dias naquelas com regimes diários de 100 e 50\% cc (Fig. 3A). 

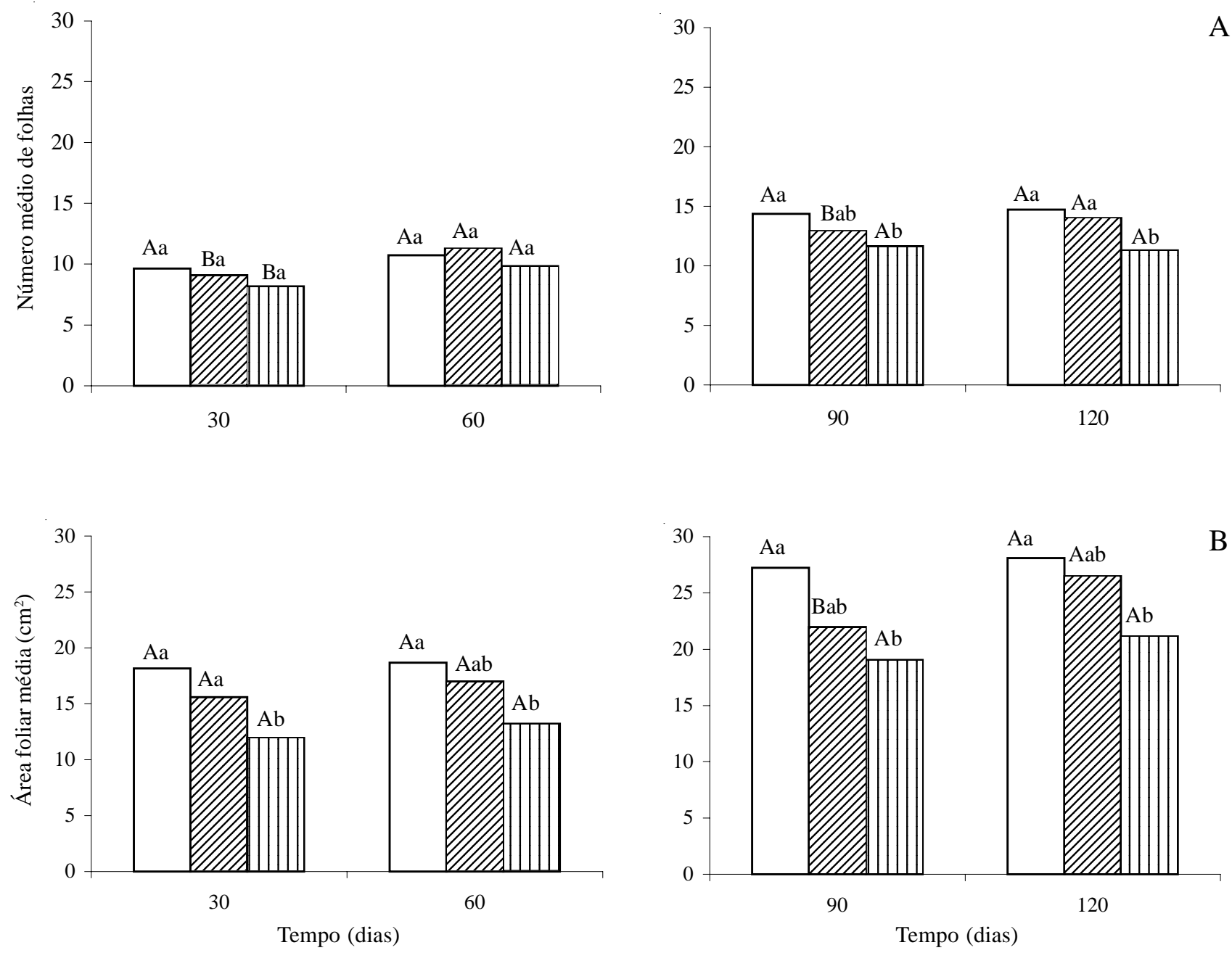

Figura 3. Número médio de folhas (A) e área foliar média (B) das plantas jovens de Tabebuia aurea (Manso) Benth \& Hook f. ex S. Moore, cultivadas em casa de vegetação sob três regimes hídricos: 100, 50 e 25\% da capacidade de campo (cc). Análise estatística realizada separadamente para os dois volumes de solo utilizados: $6 \mathrm{~kg}$ ( 30 e 60 dias) e $12 \mathrm{~kg}$ ( 90 e 120 dias). Letras iguais não diferem entre os tratamentos (minúsculas) e períodos (maiúsculas) pelo teste de Tukey à 5\% de probabilidade. $\square 100 \% \mathrm{cc} ; \square 50 \% \mathrm{cc} ; \square 25 \% \mathrm{cc}$.

Nos dados de área foliar média $\left(\mathrm{cm}^{2}\right)$, evidenciou-se a partir dos 30 dias redução acentuada nas plantas com suprimento hídrico diário de $25 \%$ cc, em relação ao tratamento $100 \%$ cc. Os valores médios das plantas com $50 \%$ cc, após 30 dias de diferenciação, foram sempre intermediários entre 100 e $25 \%$ cc (Fig. 3B).

Barbosa (1991) e Barros \& Barbosa (1995) verificaram redução significativa no número de folhas e na área foliar total das plantas jovens de Anadenanthera macrocarpa e Acacia farnesiana a partir do quarto e terceiro meses, respectivamente, no tratamento sob estresse hídrico. Kozlowski et al. (1991) comentaram que um dos efeitos mais drásticos do estresse hídrico é a redução na área foliar, levando a um decréscimo na fotossíntese e, conseqüentemente, no crescimento.
Peso seco das partes aérea e subterrânea - Para todas as variáveis analisadas não houve diferenças significativas entre os tratamentos nos dois primeiros meses (Tab. 4).

Aos 90 dias foram verificadas diferenças para os três tratamentos, em relação à matéria seca das folhas, parte aérea e total das plantas. No tratamento com $25 \%$ cc, o estresse hídrico reduziu também os pesos do caule e parte subterrânea. Com relação aos 120 dias, verificou-se diferenças significativas em todas as variáveis analisadas no tratamento com $25 \%$ cc.

O peso da matéria seca da parte aérea foi superior ao da parte subterrânea nos três tratamentos até os 90 dias; no entanto, aos 120 dias observou-se ligeiro aumento da parte subterrânea em relação à aérea. Isto se deve ao espessamento observado, na planta jovem, iniciando do hipocótilo para o sistema radicular, a partir 
Tabela 4. Peso da matéria seca (folha, caule, parte aérea, parte subterrânea e total) e razão parte subterrânea/aérea das plantas jovens de Tabebuia aurea (Manso) Benth. \& Hook f. ex S. Moore mantidas por 30, 60, 90 e 120 dias em casa de vegetação e irrigadas com 100, 50 e $25 \%$ da capacidade de campo (cc).

\begin{tabular}{|c|c|c|c|c|c|c|c|}
\hline \multirow[b]{2}{*}{$\begin{array}{l}\text { Avaliações } \\
\text { (dias) }\end{array}$} & \multirow[b]{2}{*}{$\begin{array}{c}\text { Tratamento } \\
\text { (\% da cc) }\end{array}$} & \multicolumn{6}{|c|}{ Peso da matéria seca $(\mathrm{g})$} \\
\hline & & Folha & Caule & Parte aérea & Parte subterrânea & Total & $\begin{array}{c}\text { Parte subterrânea/ } \\
\text { aérea }\end{array}$ \\
\hline \multirow{3}{*}{30} & 100 & $1,21 \mathrm{Aa}$ & $0,60 \mathrm{Ba}$ & $1,86 \mathrm{Ba}$ & $0,90 \mathrm{Ba}$ & $2,77 \mathrm{Ba}$ & $0,50 \mathrm{Ba}$ \\
\hline & 50 & $1,03 \mathrm{Aa}$ & $0,59 \mathrm{Ba}$ & $1,68 \mathrm{Ba}$ & $0,97 \mathrm{Ba}$ & $2,65 \mathrm{Ba}$ & $0,60 \mathrm{Ba}$ \\
\hline & 25 & $0,63 \mathrm{Aa}$ & $0,41 \mathrm{Aa}$ & $1,08 \mathrm{Aa}$ & $0,57 \mathrm{Aa}$ & $1,65 \mathrm{Aa}$ & $0,54 \mathrm{Ba}$ \\
\hline \multirow{3}{*}{60} & 100 & $1,58 \mathrm{Aa}$ & $1,35 \mathrm{Aa}$ & $3,05 \mathrm{Aa}$ & $2,29 \mathrm{Aa}$ & $5,35 \mathrm{Aa}$ & 0,77 Aa \\
\hline & 50 & $1,50 \mathrm{Aa}$ & $1,31 \mathrm{Aa}$ & $2,88 \mathrm{Aa}$ & $2,25 \mathrm{Aa}$ & $5,12 \mathrm{Aa}$ & $0,78 \mathrm{Aa}$ \\
\hline & 25 & $0,93 \mathrm{Aa}$ & $0,90 \mathrm{Aa}$ & $1,90 \mathrm{Aa}$ & $1,31 \mathrm{Aa}$ & $3,21 \mathrm{Aa}$ & $0,69 \mathrm{Aa}$ \\
\hline \multirow{3}{*}{90} & 100 & 3,53 Aa & $3,68 \mathrm{Ba}$ & $7,26 \mathrm{Ba}$ & $5,86 \mathrm{Ba}$ & $13,13 \mathrm{Ba}$ & $0,83 \mathrm{Ba}$ \\
\hline & 50 & $2,64 \mathrm{Bb}$ & $2,52 \mathrm{Bb}$ & $5,21 \mathrm{Bb}$ & $4,76 \mathrm{Ba}$ & $9,97 \mathrm{Bb}$ & $0,93 \mathrm{Ba}$ \\
\hline & 25 & $1,70 \mathrm{Ac}$ & $1,98 \mathrm{Ab}$ & $3,73 \mathrm{Ac}$ & $3,23 \mathrm{Bb}$ & $6,95 \mathrm{Bc}$ & $0,87 \mathrm{Ba}$ \\
\hline \multirow{3}{*}{120} & 100 & $3,71 \mathrm{Aa}$ & 4,63 Aa & $8,38 \mathrm{Aa}$ & $8,58 \mathrm{Aa}$ & $16,96 \mathrm{Aa}$ & $1,03 \mathrm{Ab}$ \\
\hline & 50 & 3,30 Aa & $4,50 \mathrm{Aa}$ & $7,85 \mathrm{Aa}$ & $8,44 \mathrm{Aa}$ & $16,29 \mathrm{Aa}$ & 1,09Aab \\
\hline & 25 & $1,81 \mathrm{Ab}$ & $2,42 \mathrm{Ab}$ & $4,27 \mathrm{Ab}$ & $5,08 \mathrm{Ab}$ & $9,35 \mathrm{Ab}$ & $1,20 \mathrm{Aa}$ \\
\hline
\end{tabular}

Letras minúsculas comparam tratamentos dentro de cada período e letras maiúsculas comparam tratamentos dentro de períodos diferentes, ao nível de $5 \%$ de probabilidade.

dos 60 dias, evidenciando-se com maior nitidez ao final do experimento (Fig. 4). Souza (1994) estudando o desenvolvimento morfo-anatômico da planta jovem da espécie em estudo, ocorrente em uma área do Cerrado de São Paulo, constatou a presença de xilopódio, órgão lenhoso, bastante característico desse ecossistema.

Esse comportamento de maior investimento da parte aérea nos primeiros meses justifica-se pelo fato dessa espécie ocorrer em áreas de Caatinga próximas às margens de riachos e rios, conseqüentemente suas raízes não sofrem muito o efeito do déficit hídrico, daí a maior alocação de matéria para os órgãos aéreos. Barbosa \& Prado (1991) e Barbosa et al. (2000) registraram resultados semelhantes para a espécie Parkinsonia aculeata ocorrente na Caatinga, nas mesmas condições de T. aurea.

Alocação de biomassa para os diferentes orgãos vegetativos - A alocação de biomassa nos diferentes órgãos das plantas praticamente não diferiu entre os tratamentos durante o período experimental (Tab. 5). Verificou-se diferenças significativas nos três tratamentos aos 30 dias, na alocação de biomassa para as folhas, e aos 30 e 90 dias para o caule. Nas folhas, o maior incremento foi para o tratamento $100 \% \mathrm{cc}$ $(43,53 \%)$, seguido do tratamento com $50 \%$ cc $(38,14 \%)$ e $25 \%$ cc $(37,80 \%)$, não havendo diferenças significativas entre os dois últimos.

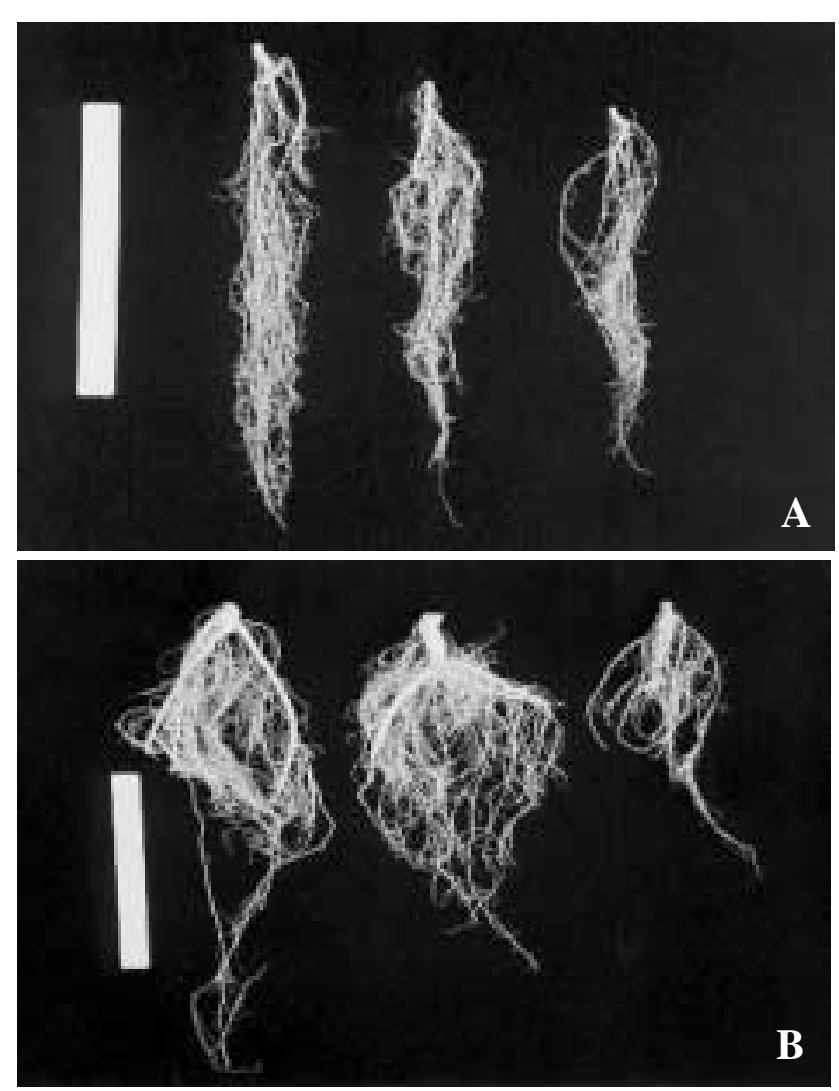

Figura 4. Aspecto da parte subterrânea das plantas jovens de Tabebuia aurea (Manso) Benth. \& Hook f. ex S. Moore, mantidas por 30 (A) e 120 (B) dias de cultivo em casa de vegetação. Irrigadas com 100,50 e $25 \%$ da capacidade de campo, seguindo a ordem da esquerda para direita $($ Escala $=20 \mathrm{~cm})$. 
Tabela 5. Alocação de biomassa durante o crescimento inicial das plantas jovens de Tabebuia aurea (Manso) Benth. \& Hook f. ex S. Moore, mantidas por 30, 60, 90 e 120 dias em casa de vegetação e irrigadas com 100, 50 e $25 \%$ da capacidade de campo (cc).

\begin{tabular}{|c|c|c|c|c|c|}
\hline \multirow{2}{*}{$\begin{array}{c}\text { Avaliações } \\
\text { (dias) }\end{array}$} & \multirow{2}{*}{$\begin{array}{l}\text { Tratamento } \\
\text { (\% da cc) }\end{array}$} & \multicolumn{4}{|c|}{ Percentagem da alocação de biomassa } \\
\hline & & Raízes & Folhas & Caules & Parte aérea \\
\hline \multirow{3}{*}{30} & 100 & $32,83 \mathrm{Ba}$ & $43,53 \mathrm{Aa}$ & $21,68 \mathrm{Bb}$ & $67,17 \mathrm{Aa}$ \\
\hline & 50 & $37,19 \mathrm{Ba}$ & $38,14 \mathrm{Ab}$ & $22,22 \mathrm{Bb}$ & $62,81 \mathrm{Aa}$ \\
\hline & 25 & $34,72 \mathrm{Ba}$ & $37,80 \mathrm{Ab}$ & $24,89 \mathrm{Ba}$ & $65,28 \mathrm{Aa}$ \\
\hline \multirow{3}{*}{60} & 100 & 43,04 Aa & $29,51 \mathrm{Ba}$ & $25,37 \mathrm{Aa}$ & $56,97 \mathrm{Ba}$ \\
\hline & 50 & $43,81 \mathrm{Aa}$ & $29,25 \mathrm{Ba}$ & $25,89 \mathrm{Aa}$ & $56,19 \mathrm{Ba}$ \\
\hline & 25 & $40,69 \mathrm{Aa}$ & $29,02 \mathrm{Ba}$ & $28,04 \mathrm{Aa}$ & $59,31 \mathrm{Ba}$ \\
\hline \multirow{3}{*}{90} & 100 & $44,92 \mathrm{Ba}$ & $26,87 \mathrm{Aa}$ & $27,82 \mathrm{Ab}$ & $55,08 \mathrm{Aa}$ \\
\hline & 50 & $47,64 \mathrm{Ba}$ & $25,99 \mathrm{Aa}$ & $25,78 \mathrm{Ab}$ & $52,36 \mathrm{Aa}$ \\
\hline & 25 & $44,92 \mathrm{Ba}$ & $24,50 \mathrm{Aa}$ & $28,61 \mathrm{Aa}$ & $53,63 \mathrm{Aa}$ \\
\hline \multirow{3}{*}{120} & 100 & $50,45 \mathrm{Aa}$ & $21,76 \mathrm{Ba}$ & $27,48 \mathrm{Aa}$ & $49,55 \mathrm{Ba}$ \\
\hline & 50 & $51,81 \mathrm{Aa}$ & $20,32 \mathrm{Ba}$ & $27,61 \mathrm{Aa}$ & $48,20 \mathrm{Ba}$ \\
\hline & 25 & $53,90 \mathrm{Aa}$ & $19,54 \mathrm{Ba}$ & $26,13 \mathrm{Ba}$ & $46,09 \mathrm{Ba}$ \\
\hline
\end{tabular}

Letras minúsculas comparam tratamentos dentro de cada período e letras maiúsculas comparam tratamentos dentro de períodos diferentes, ao nível de $5 \%$ de probabilidade.

Nos caules, aos 30 e 90 dias, a maior percentagem de biomassa foi alocada nas plantas com $25 \%$ cc, cujos valores foram de $24,89 \%$ e $28,61 \%$, respectivamente.

Analizando-se o percentual de biomassa alocada para as partes aérea (folha + caule) e subterrânea, nos diferentes tratamentos hídricos, verificou-se que nos três primeiros meses a alocação foi maior para a parte aérea e no último mês, para a parte subterrânea. Ao analisar-se separadamente os diferentes órgãos, observou-se, também aos 120 dias, que maior alocação foi para a parte subterrânea e, aos 30 dias, para as folhas.

Em plantas jovens de espécies da Caatinga foram registradas maior alocação de biomassa para a parte subterrânea em Anadenanthera macrocarpa e Senna occidentale (Barbosa 1991; Barbosa et al. 2000); caules em Jatropha urens L., Jatropha polhiana L. e Parkinsonia aculeata L. (Ferri 1955; Barros \& Barbosa 1995; Barbosa et al. 2000); folhas em Senna martiana, Mimosa caesalpiniifolia Benth. e Tabebuia aurea (Barbosa et al. 2000; Santiago 2000; Silva 2002).

Silva \& Silva (1974) e Barbosa (1980) comprovaram a natureza radicular e presença de tuberosidade, com reserva de água e amido, nas espécies Spondias tuberosa A. Cam. e Anadenanthera macrocarpa, respectivamente. A alocação de biomassa direcionada para um determinado órgão pode ser atribuída às diferenças genotípicas inerentes às espécies.
Densidade estomática - As folhas jovens de T. aurea apresentaram, nas três regiões analisadas, maior número de estômatos na superfície abaxial (inferior), consideradas hipoestomáticas, com estômatos do tipo anomocítico, variando de 119,63 a 155,19 estômatos por $\mathrm{mm}^{2}$. Diferenças significativas foram registradas apenas na região apical para os tratamentos de 50 e $25 \%$ cc, em relação ao tratamento $100 \%$ cc, aos 120 dias (Tab. 6). Na superfície adaxial (superior) encontrou-se esporadicamente 1 ou 2 estômatos por $\mathrm{mm}^{2}$, próximos à nervura principal. Valente $\&$ Carvalho (1973) trabalhando com plântulas de Aspidosperma pyrifolium (Apocynaceae), espécie característica da Caatinga, detectaram comportamento semelhante ao observado para a espécie em estudo, registrando média inferior a 47,8 estômatos por $\mathrm{mm}^{2}$ na superfície abaxial e raros na superfície adaxial.

$\mathrm{O}$ estresse hídrico fornecido às plantas jovens, nos tratamentos 50 e $25 \%$ cc, provocou aumento do número de estômatos nas três regiões das folhas aos 30 e 60 dias, e diminuição aos 90 e 120 dias, comparadas ao tratamento $100 \% \mathrm{cc}$. Essa tendência evidenciou-se mais nas plantas jovens sob tratamento de $25 \%$ cc em relação aos $50 \%$ cc. Resultados significativos foram observados, apenas, na região apical para $25 \%$ cc, com relação ao tratamento $100 \%$ cc, aos 120 dias.

Campos (1991), ao estudar o balanço hídrico de cinco espécies lenhosas adultas, nas Caatingas de Pernambuco, determinou a densidade estomática e 
Tabela 6. Densidade estomática $\left(\mathrm{mm}^{2}\right)$ das regiões apical, mediana e basal das folhas de plantas jovens de Tabebuia aurea (Manso) Benth. \& Hook f. ex S. Moore, mantidas por 30, 60, 90 e 120 dias em casa de vegetação e irrigadas com 100, 50 e $25 \%$ da capacidade de campo (cc).

\begin{tabular}{ccccc}
\hline \multirow{2}{*}{$\begin{array}{c}\text { Avaliações } \\
\text { (dias) }\end{array}$} & $\begin{array}{c}\text { Tratamento } \\
\text { (\% da cc) }\end{array}$ & Apical & Mediana & Basal \\
\cline { 3 - 5 } 30 & 100 & $127,88 \mathrm{a}$ & $126,44 \mathrm{a}$ & $122,06 \mathrm{a}$ \\
& 50 & $134,50 \mathrm{a}$ & $135,75 \mathrm{a}$ & $125,25 \mathrm{a}$ \\
& 25 & $143,00 \mathrm{a}$ & $141,63 \mathrm{a}$ & $129,36 \mathrm{a}$ \\
60 & 100 & $132,81 \mathrm{a}$ & $129,94 \mathrm{a}$ & $127,06 \mathrm{a}$ \\
& 50 & $133,88 \mathrm{a}$ & $131,13 \mathrm{a}$ & $122,13 \mathrm{a}$ \\
& 25 & $137,81 \mathrm{a}$ & $143,06 \mathrm{a}$ & $127,19 \mathrm{a}$ \\
90 & 100 & $150,63 \mathrm{a}$ & $148,88 \mathrm{a}$ & $140,31 \mathrm{a}$ \\
& 50 & $139,56 \mathrm{a}$ & $135,25 \mathrm{a}$ & $128,00 \mathrm{a}$ \\
& 25 & $129,50 \mathrm{a}$ & $129,19 \mathrm{a}$ & $119,63 \mathrm{a}$ \\
\multirow{2}{*}{120} & 100 & $155,19 \mathrm{a}$ & $136,06 \mathrm{a}$ & $145,56 \mathrm{a}$ \\
& 50 & $136,31 \mathrm{ab}$ & $129,81 \mathrm{a}$ & $141,56 \mathrm{a}$ \\
& 25 & $119,88 \mathrm{~b}$ & $121,31 \mathrm{a}$ & $135,94 \mathrm{a}$ \\
\hline
\end{tabular}

Médias seguidas pelas mesma letras nas colunas e dentro de um mesmo período não diferem entre si pelo teste de Tukey ao nível de $5 \%$ de probabilidade.

encontrou uma variação de 40 a 50 estômatos por mm². Três espécies apresentaram estômatos nas duas superfícies (anfiestomáticas) Jatropha mollissima Pohl., Prosopis juliflora D.C. e Mimosa hostilis, e duas na superfície inferior (abaxial) Caesalpinia pyramidalis Tul. e Aspidosperma pyrifolium. Nas duas primeiras anfiestomáticas, a autora registrou maior número de estômatos na superfície adaxial. Santos \& Grisi (1976), ao estudarem a anatomia foliar de oito espécies lenhosas adultas da Caatinga, identificaram cinco hipoestomáticas (Caesalpinia pyramidalis, Schinopsis brasiliensis Engl., Zizyphus joazeiro Mart., Aspidosperma pyrifolium e Bursera leptophloes (Mart.) Engl.) e três anfiestomáticas (Cnidoscolus phyllacanthus Mart., Cassia martiana Benth. e Maytenus rigida Mart.), com estômatos do tipo anomocítico, o mesmo tipo apresentado pela espécie em estudo.

Barbosa (1983) registrou para Amburana cearensis (Fr. All.) A.C. Smith. folhas hipoestomáticas, estômatos anomocíticos, situados em depressão, diferindo das espécies estudadas por Santos \& Grisi (1976) cujos estômatos estavam situados ao mesmo nível das células epidérmicas em todas as espécies estudadas, com exceção de Aspidosperma pyrifolium, com estômatos encontrados acima do nível das células epidérmicas.
O número e a localização dos estômatos são importantes como respostas à baixa disponibilidade hídrica (Fahn \& Cutler 1992). Fahmy (1997), ao estudar 20 espécies perenes, caméfitas, não suculentas dos desertos do Egito, verificou que todas possuiam estômatos em ambas as superfícies (anfiestomáticas); a densidade na superfície superior variou de 52 a 426 estômatos por $\mathrm{mm}^{2} \mathrm{e}$ na inferior, de 42 a 373 estômatos por $\mathrm{mm}^{2}$. Fahn \& Cutler (1992) constataram que estômatos nas duas superfícies tendiam a ser mais comuns em folhas espessas e em plantas de hábitats xéricos. Mott et al. (1982) observaram que vegetais com grande copa fotossintética apresentavam vantagens, quando cresciam em regiões com alta radiação solar e rápidas flutuações na disponibilidade hídrica do solo. Esses autores comentaram que em Ambrosia cordifolia (A. Gray) Payne, planta do deserto de Tucson, Arizona, ao crescerem em áreas abertas, sujeitas a forte luminosidade, apresentavam folhas com densidade estomática de 133 a 390 por mm², $^{2}$ para as superfícies superior e inferior, respectivamente. Contudo, as folhas daquela espécie, crescendo em ambientes sombreados, não mostraram estômatos na superfície superior e registraram para a superfície inferior densidade estomática média de 305 estômatos por $\mathrm{mm}^{2}$.

\section{Agradecimentos}

À Empresa Pernambucana de Pesquisa Agropecuária (IPA), por armazenar as sementes; ao Pesquisador Venézio Felipe dos Santos, pelas análises estatísticas.

\section{Referências bibliográficas}

Andrade, A.C.S.; Ramos, F.N.; Souza, A.F.; Loureiro, M.B. \& Bastos, R. 1999. Flooding effects in seedlings of Cytharexyllum myrianthum Cham. and Genipa americana L.: responses of two neotropical lowland tree species. Revista Brasileira de Botânica 22(2): 281-285.

Andrade-Lima, D. 1989. Pp.188-191. Plantas das Caatingas. Academia Brasileira de Ciências. Rio de Janeiro, RJ.

Arasaki, F. \& Felippe, G.M. 1991. Crescimento inicial e conteúdo de açúcares soluveis em Kielmeyera coriaceae. Hoehnea 18(2): 171-179.

Barbosa, D.C.A. 1980. Estudos ecofisiológicos em Anadenanthera macrocarpa (Benth.) Brenan. Aspecto da germinação e crescimento. Tese de Doutorado. Universidade de São Paulo, São Paulo.

Barbosa, D.C.A. 1991. Crescimento de Anadenanthera macrocarpa (Benth.) Brenan. (LeguminosaeMimosoideae). Phyton 52(1): 51-62. 
Barbosa, D.C.A. \& Prado M.C.G. 1991. Quantitative analysis of the growth of Parkinsonia aculeata L. in a greenhouse. Phyton 52(1): 17-26.

Barbosa, D.C.A.; Nogueira, R.J.M.C. \& Melo Filho, P.A. 2000. Comparative studies of growth in three species of "Caatinga" submitted to water stress. Phyton 69: 45-50.

Barbosa, M.C.A. 1983. Alguns aspectos morfológicos da folha de Amburana cearensis (Fr. All.) A.C. Smith., ocorrente em áreas de agreste e Caatinga do Nordeste. Brasil Florestal 53: 42-48.

Barros, L.M. \& Barbosa, D.C.A. 1995. Crescimento de Acacia farnesiana (L.) Willd. em casa de vegetação. Phyton 57(2): 179-191.

Brighenti, A.M.; Silva, J.F.; Lopes, N.F.; Cardoso, A.A. \& Ferreira, L.R. 1993. Crescimento e partição de assimilados em losna. Revista Brasileira de Fisiologia Vegetal 5(1): 41-45.

Campos, M.A.L. 1991. Aspectos morfo-fisiológico de plantas da Caatinga, durante períodos úmido e de estresse hídrico. Dissertação de Mestrado. Universidade Federal Rural de Pernambuco, Recife.

Corrêa, M.P. 1984. Dicionário das plantas úteis do Brasil e das exóticas cultivadas. Imprensa Nacional. v. 3. Rio de Janeiro.

EMBRAPA 1996. Núcleo Tecnológico para Informática SWNTIA/EMBRAPA.

Fahn, A. \& Cutler, D.F. 1992. Xerophytes. Gebrüder Borntraeger. Berlin, Stuttgart.

Fahmy, G.M. 1997. Leaf anatomy and its relation to the ecophysiology of some non-succulent desert plants from Egypt. Journal of Arid Environments 36: 499-525.

Feliciano, A.L.P. 1989. Estudo da germinação de sementes e desenvolvimento de muda, acompanhado de descrições morfológicas, de dez espécies arbóreas ocorrentes no semi-árido nordestino. Dissertação de Mestrado. Universidade Federal de Viçosa, Viçosa.

Ferri, M.G. 1955. Contribuição ao desenvolvimento da ecologia do cerrado e da Caatinga; estudo comparativo da economia d'água de sua vegetação. Boletim da Faculdade de Filosofia e Ciências, São Paulo 12(195): $1-170$.

Gama, N.S. 1992. Estudos ecofisiológicos em Bauhinia cheilantha (Bong.) Steud., (Leguminosae, caesalpinoidea) na região semi-árida do Estado de Alagoas. Dissertação de Mestrado. Universidade Federal Rural de Pernambuco, Recife.

Garwood, N.C. 1996. Functional morphological of tropical tree seedling. Pp. 59-138. In: The ecology of tropical forest tree seedlings. Ed. M.D. SWAINE. v. 17.

Gentry, A.H. 1992. Monograh 25 (II) Bignoniacea - Part II (Tribe Tecomeae). Pp. 144-145. Flora Neotropica. The New York Botanical Garden, New York.

Godoy, S.M.A. \& Felippe, G.M. 1992. Crescimento inicial de Qualea cordata, uma árvore dos cerrados. Revista Brasileira de Botânica 15(1): 23-30.

Hamburgo-Alves, J.L. 1975. Anatomia do desenvolvimento de Jacaranda brasiliana (Lam.) Pers. - (Bignoniaceae). Tese de Doutorado. Instituto de Biociências da Universidade de São Paulo, São Paulo.
Handro, W. 1969. Contribuição ao estudo da unidade de dispersão e da plântula de Andira humilis Mart. ex Benth. (Leguminosae - Lotoideae). Boletim da Faculdade de Filosofia, Ciências e Letras da Universidade de São Paulo. n. 349. Botânica n. 27.

Hsiao, T.C. 1973. Plant response to water stress. Annual Review of Plant Physiology 24: 519-570.

Kozlowski, T.T.; Kramer, P.J. \& Pallardy, S.G. 1991. Water stress. Pp. 248-302. In: The physiological ecology of woody plants. Academic Press, New York.

Larcher, W. 2000. Ecofisiologia Vegetal. Rima Artes e Textos, São Carlos.

Lorenzi, H. 1992. Árvores Brasileiras - Manual de identificação e cultivo de plantas arbóreas nativas do Brasil. Ed. Plantarum Ltda., Nova Odessa.

Mansur, R.J.C.N. \& Barbosa D.C.A. 2000. Comportamento fisiológico em plantas jovens de quatro espécies lenhosas da Caatinga submetidas a dois ciclos de estresse hídrico. Phyton 68: 97-106.

Melo, M.D. 1995. Morfo-anatomia comparativa de indivíduos distintos de Tabebuia aurea (Manso) Benth. \& Hook. f. ex S. Moore (Bignoniaceae). Dissertação de Mestrado. Universidade Federal Rural de Pernambuco, Recife.

Moraes, J.M.F. 2002. Efeitos de diferentes níveis de água na germinação e no crescimento de Myracrodruon urundeuva Allemão (Anacardiaceae). Dissertação de Mestrado. Universidade Federal de Pernambuco, Recife.

Mott, K.A.; Gibson, A.C. \& O'Leary, J.W. 1982. The adaptive significance of amphistomatic leaves. Plant, Cell and Environment 5(4): 455-460.

Nardoto, G.B.; Souza, M.P. \& Franco, A.C. 1998. Estabelecimento e padrões sazonais de produtividade de Kielmeyera coriacea (Spr) Mart. nos cerrados do Planalto Central: efeitos do estresse hídrico e sombreamento. Revista Brasileira Botânica 21(3): 313-319.

Nogueira, R.J.M.C. 1997. Expressões fisiológicas em aceroleiras (Malpighia emarginata D.C.) em condições adversas. Tese de Doutorado. Universidade Federal de São Carlos, São Carlos.

Paulilo, M.T.S. \& Felippe, G.M. 1994. Contribuição dos cotilédones e partição de matéria durante o crescimento inicial de Qualea grandiflora Mart. (Vochysiaceae). Revista Brasileira de Botânica 17(2): 87-91.

Paulilo, M.T.S. \& Felippe, G.M. 1995. Respostas de plântulas de Qualea grandiflora Mart., uma espécie arbórea do cerrado, à adição de nutrientes minerais. Revista Brasileira de Botânica 18(1): 109-112.

Paulilo, M.T.S.; Felippe, G.M. \& Dale J.E. 1993. Crescimento inicial de Qualea Grandiflora. Revista Brasileira de Botânica 16(1): 37-46.

Perez, S.C.J.G.A. 1995. Crescimento e resistência à seca da algarobeira (Prosopis juliflora Sw. D.C.) cultivada em solo de cerrado, com ou sem adubo orgânico. Pesquisa Agropecuária Brasileira 30(5): 595-604.

Poggiani, F. 1971. Estudo do crescimento e da atividade AIA-oxidásica em algumas espécies do cerrado. Pp. 201-211. In: M.G. Ferri (ed.). III Simpósio sobre o cerrado. Ed. Edgar Blucher e EDUSP, São Paulo. 
Poggiani, F. 1973. Aspectos do crescimento e do metabolismo auxínico de espécies do cerrado. Tese de Doutorado, Universidade de São Paulo, São Paulo.

Poggiani, F.; Simões, I.W. \& Mendes-Filho, I.M.A. 1981. Utilização de espécies florestais de rápido crescimento na recuperação de áreas degradadas. Piracicaba: ESALQ, Instituto de Pesquisas e Estudos Florestais, Departamento de Silvicultura. Série Técnica 2(4): 1-25.

Rizzini, C.T. 1997. Tratado de Fitogeografia do Brasil. Âmbito Cultural Edições Ltda, Rio de Janeiro.

Sampaio E.V.S.B. 1995. Overview of the Brazilian Caatinga. Pp. 35-63. In: S.H. Bullock; H.A. Moonev \& E. Medina (eds.). Seasonally dry Tropical Forest. Cambridge, New York.

Santiago, A.M.P. 2000. Aspectos do crescimento do sabiá (Mimosa caesalpiniifolia Benth.) em função da disponibilidade de água no solo. Dissertação de Mestrado. Universidade Federal Rural de Pernambuco, Recife.

Santos, A.V.P. \& Grisi, B.M. 1976. Anatomia foliar ecológica de algumas plantas da Caatinga. Revista Brasileira de Biologia 56(4): 773-787.

Sassaki, R.M. \& Felippe, G.M. 1992. Remoção dos cotilédones e desenvolvimento inicial de Dalbergia miscolobium. Revista Brasileira de Botânica 15(1): 5-16.

Silva, A.A.Q. \& Silva, M.A.G.O. 1974. Observações morfológicas e fisiológicas sobre Spondias tuberosa A. Cam. Pp. 5-15. Anais do XXV Congresso Nacional de Botânica. Mossoró, RN.
Silva, E.C. 2002. Ecofisiologia de quatro espécies lenhosas ocorrentes no Nordeste, submetidas a estresse hídrico. Dissertação de Mestrado. Universidade Federal Rural de Pernambuco, Recife.

Sousa, A.V.G. 1994. Morfologia, desenvolvimento e anatomia de Tabebuia caraiba (Mart.) Bur. - Bignoniaceae. Tese de Doutorado. Universidade de São Paulo, São Paulo.

Souto, M.S. 1996. Caracteres morfológicos de frutos e sementes, poder germinativo (e crescimento) de plantas jovens de 6 espécies de Leguminosae da Caatinga de Alagoinha, PE. Dissertação de Mestrado. Universidade Federal de Pernambuco, Recife.

Teixeira, P.G.; Carvalho, M.A.M.; Zaidan, L.B.P. \& Klein, A.L. 1997. Effect of mineral nutrients on growth and fructan contents in plants of Vernonia herbacea. Revista Brasileira de Fisiologia Vegetal 9(2): 89-96.

UTHSCSA. 1997. Image Tool. University of Texas Health Science Center. San Antonio, Texas.

Valente, M.C. \& Carvalho, L.D’A.F. 1973. Plantas da Caatinga I - Apocynaceae. Anatomia e desenvolvimento de Aspidosperma pyrifolium Mart. var. molle Muell. Arg. - "Pereiro". Revista Brasileira de Biologia 33(2): 285-301.

Vidal, M.C.; Stacciarini-Seraphin, E. \& Câmara, H.H.L.L. 1999. Crescimento de plântulas de Solanum lycocarpum St. Hil. (Lobeira) em casa de vegetação. Acta Botanica Brasilica 13(3): 271-274. 
